\title{
DIDÁCTICA DE LA LENGUA Y LA LITERATURA: LA UTOPÍA NECESARIA PARA EL BIENESTAR PERSONAL
}

\author{
Amando López Valero \\ Eduardo Encabo Fernández \\ Carmelo Moreno Muñoz \\ Universidad de Murcia
}

\begin{abstract}
RESUMEN. El propósito del presente trabajo es poner de manifiesto un punto de vista particular acerca de la importancia del lenguaje y de su enseñanza. Vivimos en una época de incertidumbre donde los cambios son muy veloces, y el lenguaje también se ve afectado por esas transformaciones. La cuestión es si los cambios ayudan al bienestar personal o al disminuir el conocimiento del lenguaje se tiene un peor conocimiento de la realidad.

Desde este artículo pretendemos exponer las razones por las cuales defendemos la importancia de la Didáctica de la Lengua y la Literatura en la formación de las personas y por encima de todo, cómo influye en el bienestar personal. Tal vez debamos eliminar el adjetivo de utópica que adjudicamos a la educación lingüística y literaria que defendemos y otorgarle el de realidad que vamos a alcanzar a través de un planteamiento crítico y comunicativo.
\end{abstract}

ABSTRACT. The aim of this work is to outline a point of view about the importance of language and its teaching. We are living in an uncertain age where changes are faster than before, and language is affected by that transformations too. The question is if changes help welfare or we have a worse knowledge of reality.

From this article we pretend to expound the reasons to defend the importance of Language and Literature Teaching and, above all, how it helps welfare. Maybe we should rename the utopian linguistic and literary education and name it as a reality we can obtain throughout a critical and communicative focus.

\section{Introducción}

A lo largo de la historia de la humanidad en muchas ocasiones se ha cuestionado cuál es el motivo de que las personas tengan que transitar por el camino de la vida; es más, también se ha sopesado si el ser humano era el eje de la existencia o había razones suprahumanas o divinas que velaban o dirigían sus actuaciones. Por encima de estas disquisiciones pensamos que se encuentra el hecho de buscar la felicidad humana, aspecto que se debe traducir en el bienestar personal, en una sensación de 
seguridad y buen hacer que facilite un adecuado tránsito a lo largo de la vida. Siendo ésta nuestra principal preocupación, es pertinente indagar en cuáles son los instrumentos que van a servir como puentes para conseguir el citado bienestar. En dicha búsqueda, por encima del resto hallamos el lenguaje; éste como capacidad exclusivamente humana se convierte en la herramienta esencial que nos permite interpretar la realidad que nos es más cercana y, de igual modo, la más lejana (Mauthner, 2001). Ahora bien, cabe cuestionarse si el lenguaje es algo que acontece de manera innata sin necesidad de que sea modificado o requiere de una depuración, en definitiva de un proceso de aprendizaje.

Si nos apoyamos en las teorías chomskianas encontraremos como punto de partida la competencia lingüística, es decir, existen unas estructuras innatas que nos van a facilitar el aprendizaje de toda una serie de manifestaciones lingüísticas que con posterioridad se pondrán a nuestro servicio. Queremos decir que, pese a que tengamos unas facultades, necesitamos de un proceso formativo adecuado para poder vivenciar todas las posibilidades que la vida nos ofrece. El lenguaje, como lente que nos facilita el acceso a la realidad, va a condicionar esa entrada en la misma: cuanto mejor sea nuestro conocimiento y utilización del lenguaje, más completa será nuestra visión de la realidad social, tanto de forma individual como colectiva. Efectuada esta precisión de ideas, para contextualizar esta pretensión de considerar los procesos de enseñanza y aprendizaje de la Lengua y la Literatura como llave de acceso al bienestar, tenemos que describir en qué tipo de sociedad van a acontecer y qué pros y contras va a conllevar esa tipología social.

\section{La educación lingüística y literaria en un mundo globalizado}

Si bien el lenguaje ha acontecido a lo largo del tiempo, su importancia y consideración ha sido diferente según las épocas. En la que a nosotros nos concierne que es la referente al siglo XXI, se halla notablemente influenciado por los avances de tipo tecnológico y ante todo por la necesidad de aquello llamado producción. Todo se ha mercantilizado y el objetivo último es el de alcanzar los máximos beneficios en todos los ámbitos que atañen a la vida. Esto trae como consecuencia que a los aspectos que requieren un sumo cuidado y dedicación como son la Lengua y la Literatura no se les pueda prestar el espacio temporal óptimo para su desarrollo. La globalización hace que no interese tanto la estética y calidad de la información, de los mensajes, como la velocidad a la cual ellos sean transmitidos. Sin ningún género de dudas esto afecta a los procesos formativos referidos a la Lengua y la Literatura, ya que la abundancia de frases muy cortas, la restricción de vocabulario o la aparición de monosílabos dentro del entramado social también tiene su reflejo en las aulas y la motivación por aprender Lengua y Literatura queda seriamente dañada. Una sociedad donde sólo preocupa lo monetario tiende a dejar de lado su vertiente cultural (Chomsky, 2001), la cual se articula a partir del lenguaje. Las diferentes muestras artísticas requieren manifestaciones lingüísticas que sirvan como vehículo para poder mostrarse a la percepción humana, ¿se estará olvidando el gusto por lo humanístico?

Si como foco principal de actuación se encuentra el hacinar más, es decir, el conseguir una mayor producción, está claro que los esquemas jerárquicos que se refieren 
a las estructuras sociales van a cambiar siguiendo un patrón de embudo, así cada vez van a ser menos las personas que lleguen a los privilegios que nos oferta la sociedad. Por tanto, no va a existir la promulgada democracia, sino que encubierto en la misma acontece un sistema de tipo autoritario (Navarro, 2002). Siendo el lenguaje el instrumento que nos ayuda a interpretar y comprender la realidad, no es de extrañar que su enseñanza esté estructurada de una forma poco flexible que se oriente hacia la especialización, a la adquisición de un léxico, de unas estructuras gramaticales y de un bagaje literario que tenga que ver con una determinada función social, sin permitir que se desarrolle el potencial que las personas puedan albergar en su interior. Asistimos pues a una transformación del lenguaje, ya que los significantes están sensiblemente cambiados: se tiende al uso desmesurado de las siglas o a la creación de nuevos códigos que en lugar de complementar a los ya existentes, poco a poco van ocupando su lugar. Con todo ello el léxico queda seriamente reducido y el uso del lenguaje muy restringido limitándose a una función instrumental, dejando de lado otras funciones como pueda ser la imaginativa o personal. Por ello, hemos llegado a una situación donde la puesta en práctica de las diferentes habilidades lingüísticas alcanza el calificativo de problema, ya que ninguna de ellas es desarrollada de una manera óptima, pues el sistema no lo está demandando; la comodidad que se establece no invita a perfeccionar dichas habilidades y por ello, el uso del lenguaje tiende a empobrecerse. De todos modos, como alternativa a esa situación se debe aportar una enseñanza de la Lengua y la Literatura que propugne la recuperación del gusto por desarrollar esas habilidades lingüísticas para lograr un mayor bienestar de las personas.

\section{El desarrollo de las habilidades lingüísticas: un desafío actual}

Un cuarteto interrelacionado constituye lo que se denomina habilidades lingüísticas: hablar, escuchar, leer y escribir. Como bien hemos mencionado en el anterior apartado su correcto y amplio uso por parte de las personas puede ser puesto en entredicho porque las características que se asocian a su desarrollo en el siglo XXI no son demasiado halagüeñas, ya que no se habla de una manera correcta en cuanto a pronunciación y un correcto uso de los términos y estructuras; tampoco se escucha de un óptimo modo ya que más bien se oye, sin apreciar el verdadero sentido de lo que las otras personas desean transmitirnos. $Y$ en lo que respecta a leer, se hace poco y sin una buena comprensión, mientras que escribir es una losa para las personas porque como consecuencia de una deficiente lectura no encuentran el referente retroalimentador para llevar a buen fin esta habilidad lingüística. El panorama es poco alentador, ya que esta situación tiene su reflejo en el entramado social, concretamente en acontecimientos de tipo interactivo donde la comunicación tiende a ser cada vez más limitada. Realmente el lenguaje está configurado para ser un instrumento de acción social (Holtgraves, 2001), es decir, pretende poner en relación a la persona con su entorno más cercano y más lejano. Si restringimos el uso de las habilidades lingüísticas, estamos empobreciendo el desarrollo del lenguaje y por ende el conocimiento de la realidad. La cuestión es que necesitamos un impulso que abogue por la utilización de las mencionadas habilidades, ya que gracias a ello la persona va a lograr una realización personal y va a ampliar sus posibilidades de expansión. 
¿En qué ámbitos es posible desarrollar las habilidades lingüísticas? Evidentemente, para que el cuarteto pueda tener una progresión integral se debe generar una situación de interactividad entre personas, ya que entornos que reduzcan cualquiera de las cuatro habilidades coartan la formación de las personas. Así, si estamos ubicados en un aula, tenemos que procurar que el alumnado y el profesorado establezcan una relación comunicativa donde se trabajen todos los aspectos, es decir a través de estrategias didácticas es factible lograr que hablar, escuchar, leer y escribir se pongan en juego, y de forma paulatina puedan ser mejoradas. De igual modo, en la familia o en los grupos de amistad es conveniente que los motivos principales de reunión e interacción no sean soportes físicos que se convierten en protagonistas -como pueda ser la televisión- ya que va a limitar el desarrollo comunicativo de las personas participantes en la situación. Pero, como bien hemos indicado, las características de la sociedad que nos acontece derivan en una realidad donde el desarrollo de las habilidades lingüísticas llega a ser todo un reto muy difícil de conseguir (López y Encabo, 2001a). Desde la Educación y, más concretamente, desde la Didáctica de la Lengua y la Literatura hay que lograr que los procesos formativos se transformen y lleguen a ser algo motivador para las personas que se hallan inmersas en los procesos de enseñanza y aprendizaje, por esa razón en el siguiente de los apartados vamos a tratar cómo estaría configurado el lugar de trabajo para que nuestra pretensión pueda ser conseguida.

\section{Un aula que dialoga es un espacio donde se construye}

Desde la redacción de estas líneas intentamos partir de la concepción de la enseñanza y del aprendizaje no como hechos reproductores y repetitivos sino como situaciones donde las personas reconstruyen y redescubren una realidad y unos conocimientos que ya existen, dotándolos de una visión personal, es decir, interiorizándolos. Por tanto, a la hora de plantear la educación, tenemos que pensar en qué tipo de conocimientos queremos que las personas manejen y adquieran. Desde nuestra óptica, deseamos que los aprendizajes memorísticos se reduzcan hasta lo indispensable ya que consideramos que son más importantes otro tipo de destrezas. Como hemos podido denotar de nuestra propia reflexión, una persona que domina su lenguaje tiene efectuada gran parte del camino hacia el aprendizaje, ya que va a poseer el instrumento que le va a facilitar el acceso. Sin ir más lejos, hemos de ser conscientes de que muchos de los saberes se encuentran escritos en libros o artículos; si una persona ejecuta de una manera correcta su habilidad lectora, no va a tener inconveniente en aproximarse y comprender qué es lo que indican esos textos. Puede suceder que para la interpretación se requiera cierto bagaje sociocultural, pero esto puede ser subsanado por la acción guiadora del profesorado que debe replantear su rol y tratar de ayudar a la mejora del alumnado, es decir, explicarle aquellas cosas que el estudiante en su proceso de aprendizaje no ha comprendido o sobre la que pretende saber más. Para llevar a buen fin este propósito es fundamental el hecho comunicativo; asentar las bases de la enseñanza sobre la acción que supone comunicar nos parece decisivo a la hora de configurar un currículo que satisfaga los intereses del alumnado (Gómez, 2000). Tenemos que recordar que la sociedad en la cual se va a insertar ese alumnado cuando finalice su periplo se va a encontrar en constante proceso de cambio, por lo que los saberes que se le otorguen a la persona tienen que ser de tipo fun- 
cional, y que no se encuentren estancados en el pasado. Por ello, los conocimientos en que se convierten las habilidades lingüísticas tienen una naturaleza atemporal, ya que únicamente necesitan moldearse a las circunstancias sociales; pero un uso correcto y asiduo de las mismas, hace que sean herramientas que posean una desmesurada utilidad.

Optar por la acción comunicativa va a suponer una transformación en la concepción de la educación, de los modelos tradicionales donde la palabra recae en la persona que a priori más conocimientos posee como es el profesorado; no se pretende restar autoridad científica al mismo; de lo que se trata es de intentar que el alumnado poco a poco, de una manera guiada, vaya llegando a establecer sus propias bases de acceso al conocimiento. Por ello, nos encontraremos en una evidente situación de mejora donde se dará más protagonismo al objeto de la educación que en realidad es que las personas adquieran una serie de destrezas para poder desenvolverse de manera óptima dentro de la sociedad.

\section{Cuando comunicarse supone el inicio de una mejora}

El esquema comunicativo es algo ya clásico dentro de las sociedades. Una persona emisora envía un mensaje a una persona receptora y ese mensaje se halla condicionado por un contexto, por un código y por un canal. Esa explicación es algo sencillo, y creemos que en ella debe fundamentarse gran parte de nuestra exposición de argumentos con el fin del inicio de la mejora, ya que consideramos que, algunos de los elementos que componen ese proceso comunicativo carecen de un carácter óptimo para que la acción comunicativa derive en una adecuada formación de las personas. Esto es, la persona receptora no posee en muchas ocasiones capacidad de respuesta ante el mensaje que le es transmitido con lo que no se produce bidireccionalidad, el contexto en muchas ocasiones es omitido, mientras que los canales cada vez son más selectivos y mecánicos y los códigos pasan a ser algo con carácter restringido. La situación descrita sería la más exacerbada, pero si nos paramos a reflexionar veremos que de forma separada esta realidad acontece en la educación, ya tenga ésta un carácter formal, no formal o informal. Según nuestra visión, los procesos de enseñanza y aprendizaje deberían comenzar su mejora a partir del replanteamiento de estas acciones comunicativas. Se trataría de procurar que los mencionados elementos se coordinaran entre sí de una manera óptima o ideal. Así, tendríamos que abogar por el enriquecimiento de los códigos que inevitablemente tiene que transcurrir por la didáctica del vocabulario para que las personas puedan manejar una "caja de herramientas" léxica que le permita adaptarse a las distintas situaciones contextuales por las que va a transitar; la complementariedad de canales también es un hecho decisivo ya que los medios tecnológicos no pueden dejar en un plano secundario al aire, en definitiva al contacto directo; y, cómo no, el contexto debe ser ampliamente conocido y adaptado a la acción comunicativa. Esto unido a la bidireccionalidad o multidireccionalidad de las situaciones comunicativas debe conferir una mejor condición para que las personas lleven a cabo los procesos de enseñanza y aprendizaje (López y Encabo, 2001b). 
La problemática surge cuando tenemos que conjuntar todas estas pautas dentro de un marco de referencia adecuado, ya que propugnar la comunicación en la enseñanza no puede quedar como un hecho aislado sino que tiene que tener su reflejo dentro de todo el devenir formativo. Por ello, dentro de la organización del currículo, en lo que respecta a la metodología de trabajo con los contenidos debe incluirse un enfoque de tipo comunicativo (Ferrada, 2001), para que podamos constatar y llevar a la práctica nuestras intenciones. Es preciso recordar que las acciones que se llevan a buen fin en la institución educativa, luego tienen su reflejo en la sociedad, y por eso es tan importante que ya desde los primeros aprendizajes se transmita al alumnado la trascendencia de poder ejecutar de una manera óptima el acto de comunicar. Con lo dicho hasta ahora es pertinente esbozar cuál sería nuestra aportación desde el área de conocimiento de Didáctica de la Lengua y la Literatura. Para ello, daremos las indicaciones pertinentes para orientar el discurrir de esta disciplina teórica que tiene su aplicación práctica en las aulas.

\section{Presupuestos básicos de la Didáctica de la Lengua y la Literatura}

Enseñar Lengua y Literatura es una tarea harto complicada y lo es por la naturaleza de los contenidos que hay que transmitir al alumnado. Pensemos que el dominio de las distintas manifestaciones del lenguaje es la llave de acceso al resto de conocimientos, además de ser la clave para la completa comprensión del mundo inteligible. La opción que conlleva basarnos en ese pensamiento es intentar no quedarnos anclados en un conocimiento específico que tiene que ver con una etapa determinada de la vida sino que aprender Lengua y Literatura tiene mucha relación con la evolución de la persona y con su adaptación a los distintos contextos sociales en los cuales se va a desenvolver. La enseñanza, la cultura y el lenguaje van a ocupar pues un mismo plano de acción (Neito, 2001) ya que existirá una interrelación entre ellos, esto es, el lenguaje es el instrumento más extendido para que la enseñanza pueda acontecer mientras que esa enseñanza tiene como misión introducir a la persona en la determinada cultura de la cual es parte y que a la vez le trasciende. Hablando de cultura y dejando un tanto de lado los contenidos de tipo cultural, en lo que respecta al halo que nos rodea tenemos que indicar que las tendencias educativas deben ir orientadas hacia el denominado aprendizaje para toda la vida -lifelong learning- (King, 1999) el cual para poder ser llevado a buen fin va a necesitar de un marco de actuación que respalde la novedosa idea que supone. Así, para poder ponerlo en práctica necesitaremos un nuevo sistema de calificaciones para todas las personas; en el área de Lengua y Literatura es muy complicado cuantificar, por tanto tendremos que basarnos en la evolución de la persona porque a la articulación del discurso no se le pueden otorgar cifras. De igual modo, necesitamos de una notable inversión en recursos que ayuden a llevar a la práctica una buena educación lingüística; de todos modos, si el profesorado es imaginativo sabrá sacar partido de muchos materiales y conocimientos que no siendo propiamente escolares sí que poseen una naturaleza susceptible de ser didáctica. El hecho de valorar la educación como hecho social va a ser trascendente para poder realizar una Didáctica de la Lengua y la Literatura que repercuta en la formación de las personas. Con esto estamos indicando que hay que respetar el hecho de que se trabajen las estructuras lingüísticas y literarias ya que el resultado que se va a 
derivar de las mismas es más importante de lo que a primera vista pueda aparentar. Un último aspecto que siempre es problemático en su tratamiento es la prolongación de la acción educativa a las familias u otros colectivos, ya que influyen sobremanera en la consolidación o erradicación de lo aprendido en las horas escolares. Lo ideal es que sirvan como complemento para que las personas sean beneficiadas.

La transposición de lo dicho hasta ahora tiene lugar en las aulas mediante la transformación de la mentalidad del profesorado; éste tiene que tratar de ir más allá de lo puramente instruccional e incidir en la formación integral del alumnado (Richards. 1998). En el área de Lengua y Literatura un ejemplo claro estaría referido a la reducción del énfasis sobre el aprendizaje de la gramática repercutiendo esto en una dedicación mayor al uso de la Lengua y a la lectura de los textos literarios. Como hemos indicado las carencias léxicas de las personas que habitan en el siglo XXI motivan que haya que buscar estrategias didácticas que suplan ese déficit. Por tanto la educación lingüística y literaria queremos basarla en la producción de textos, tanto orales como escritos, que redunden en la articulación del discurso de las personas. Se trata de plantear actividades enmarcadas en unidades temáticas que permitan al alumnado poner en juego sus habilidades lingüísticas para ir complementando las mismas con las aportaciones del colectivo que estará guiado por el profesorado. De ese modo, el aula se convierte en un lugar marcado por un clima lingüístico y literario, y se deja de lado la rigidez que implica el hecho de ceñirse al estudio exhaustivo de la gramática o de las biografías literarias (López y Encabo, 2001c).

Así pues los presupuestos básicos de esta disciplina que desde estas líneas estamos defendiendo pasan inevitablemente por la acción reflexiva del profesorado para poder llevar a la práctica una educación de las características que estamos describiendo. El colectivo docente debe plantearse de un modo crítico qué está haciendo en su práctica cotidiana (Kincheloe, 1993). Concretamente, el de Lengua y Literatura tiene que pensar si está provocando que las personas autorregulen sus usos del lenguaje o si estos están quedando atrapados entre estructuras rígidas y demasiado teóricas. El enfoque comunicativo funcional tiene que guiar la práctica en las clases de Lengua y Literatura para que el lenguaje sea algo vivo que sirva para mejorar la vida de las personas, en definitiva, para que repercuta en su bienestar.

\section{Conclusiones. ¿Utopía, necesidad o diáfana realidad?}

Llega el momento de hacer balance sobre las ideas que han sido tratadas a lo largo del texto. Sin ánimo de que se convierta en algo ampuloso queremos volver a retomar la cuestión referida a la importancia de la enseñanza de la Lengua y la Literatura. Esta reivindicación se deriva de la época incierta de la cual somos componentes; en ella, al estar los aprendizajes tan especializados y encasillados se cae en el olvido de la imaginación y por ello la institución escolar se convierte en una rémora para el alumnado, que termina por aborrecer las aulas (Trilla, 2002). Pero, la paradoja clara es que el lenguaje va a estar con la persona a lo largo de toda su vida y el ser humano necesita desarrollarse, por lo que no hay lugar para la discusión, la Didáctica de la Lengua y la Literatura pasa a ser una necesidad, la utopía necesaria ya que sin ella no habría evolución. Hay que despertar las mentes y otorgar a la Educación su papel destacado 
en el ámbito social, en definitiva, en la vida, porque como nos indica Delors (1996) el acto de educar es valioso, y no podemos dejar que ese esplendor se vaya apagando en beneficio de lo mecánico.

La alternativa a esta realidad pasa por la realización de un juicio crítico a la misma, observando qué aspectos son susceptibles de cambio y qué es necesario para conseguirlo. Desde la opción que defendemos es el consenso el mejor aliado que podemos tener, pero para lograrlo nos hace falta que acontezca el proceso comunicativo, donde se debatan los aspectos positivos y negativos de las situaciones. El lenguaje es un arma que tiene mucho poder y dependiendo de su utilización vamos a hacer que las realidades sociales se desarrollen de un modo u otro, es decir que si el lenguaje puede conducirnos al consenso también su uso puede motivar la perpetuación de las estructuras. Si esto ocurre, no estamos haciendo que el ser humano evolucione sino que estamos confiriendo poder a determinados estamentos de la sociedad actual, la cual como bien hemos indicado, tiende a la especialización y a la degeneración lingüística; con este término queremos hacer alusión a los problemas que surgen con respecto al uso de las habilidades lingüísticas. Como área de conocimiento o materia dentro de los planes de estudio, la Lengua y la Literatura posee los mismos problemas que los socialmente reflejados. La realidad es que tanto la Lengua como la Literatura se convierten en dos ajados aspectos que han sido dejados de lado dentro del contexto social; pese a su obligado uso, no se aprecia un cuidado y una evolución sino que se tiende a empobrecer su utilización. Desde la Didáctica de la Lengua y la Literatura se debe alzar la voz para propugnar un enfoque distinto de la educación lingüística y literaria que provoque la inversión de esa situación y entonces sea cuando el lenguaje sea algo vivo que esté al servicio de la vida de las personas. La reflexión por parte de los profesionales de la educación se nos antoja esencial para poder idear nuevas aportaciones que sirvan para mejorar el vocabulario, para el aumento de la extensión de las frases, para el fomento de la imaginación, en definitiva: para poder hacer un uso de la Lengua y de la Literatura acorde con las posibilidades que éstas nos ofrecen. El bienestar personal no se obtiene a través del hacinamiento, de la obtención de muchas cosas materiales, sino más bien mediante la mejora personal, y en ella tiene mucho que ver el lenguaje, ya que como venimos repitiendo a lo largo del texto es el instrumento que nos facilita el acceso y la interpretación de la realidad. ¡Cuántas cosas se quedan sin ser conocidas por la falta de utilización de una herramienta lingüística que permita comprender! Aboguemos pues por la construcción de entornos comunicativos donde las personas estén en interacción las unas con las otras, y donde el conocimiento pueda ser reconstruido con la intención de que cada persona posea la visión colectiva, pero a la vez personal, de los elementos que componen la realidad en la cual habita. Es una clara realidad que necesitamos el lenguaje para todos los aspectos de nuestra vida, ¿por qué no vamos a aprovechar las muchas posibilidades que nos ofrece? Tratemos en la medida de nuestras posibilidades de propiciar situaciones donde las habilidades lingüísticas tengan que ser puestas en práctica ya que ello favorecerá nuestra formación integral y sobre todo, nuestro bienestar. 


\section{Obras citadas}

CHOMSKY, N. (2001) El beneficio es lo que cuenta: neoliberalismo y orden global (Barcelona, Crítica).

DELORS, J. (1996) La educación o la utopía necesaria. En Informe a la UNESCO de la Comisión Internacional sobre la Educación. La educación encierra un tesoro (Madrid, Santillana/Ediciones UNESCO), 13-36.

FERRADA, D. (2001) Curriculum crítico comunicativo (Barcelona, El Roure).

GÓMEZ, I. (2000) Bases teóricas de una propuesta didáctica para favorecer la comunicación en el aula. En J. Jorba, I. Gómez y A. Prat (Eds.) Hablar y escribir para aprender (Barcelona, ICE. Universitat Autónoma de Barcelona/Síntesis), 19-28.

HOLTGRAVES, T. M. (2001) Language as social action. Social Psychology and Language use (Londres, Lawrence Erlbaum Associates).

KINCHELOE, J. L. (1993) Toward a critical politics of teacher thinking (Westport, Greenwood Publishing Group).

KING, E. (1999) Education Revised for a World in transformation. Comparative Education, 35 (2), 109-117.

LÓPEZ, A. y ENCABO, E. (2001a) El desarrollo de habilidades lingüísticas. Una perspectiva crítica (Granada, Grupo Editorial Universitario).

LÓPEZ, A. y ENCABO, E. (2001b) Mejorar la comunicación en niños y adolescentes (Madrid, Pirámide).

LÓPEZ, A. y ENCABO, E. (2001c) Heurística de la comunicación. El aula feliz (Barcelona, Octaedro).

MAUTHNER, F. (2001) Contribuciones a una crítica del lenguaje (Barcelona, Herder).

NAVARRO, V. (2002) Bienestar insuficiente, democracia incompleta: sobre lo que no se habla en nuestro país (Barcelona, Anagrama).

NEITO, S. (2001) Language, Culture and Teaching. Critical Perspectives for a New Century (London, Lawrence Erlbaum Associates).

RICHARDS, J. C. (1998) Beyond training. Perspectives on Language Teacher Education (Cambridge, University Press).

TRILLA, J. (2002) La aborrecida escuela. Junto a una pedagogía de la felicidad y otras cosas (Barcelona, Laertes). 